APPLiCATIONES MATHEMATICAE

25,4 (1999), pp. 295-299

A. S. NOWAK (Wrocław)

\title{
A GENERALIZATION OF UENO'S INEQUALITY FOR $n$-STEP TRANSITION PROBABILITIES
}

Abstract. We provide a generalization of Ueno's inequality for $n$-step transition probabilities of Markov chains in a general state space. Our result is relevant to the study of adaptive control problems and approximation problems in the theory of discrete-time Markov decision processes and stochastic games.

Let $(S, \mathcal{F})$ be a measurable space and let $P$ and $Q$ be transition probabilities from $S$ into $S$. The composition of $P$ and $Q$, denoted by $P Q$, is the transition probability defined by

$$
P Q(s, B)=\int_{S} Q(z, B) P(s, d z),
$$

where $s \in S, B \in \mathcal{F}$. For any integer $n \geq 2$, we write $Q^{n}$ to denote the $n$-step transition probability $Q Q^{n-1}$ from $S$ into $S$, induced by $Q^{1}=Q$. By $\|\cdot\|$, we denote the total variation norm in the vector space of all finite signed measures on $(S, \mathcal{F})$. Recall that if $\mu_{1}$ and $\mu_{2}$ are probability measures on $(S, \mathcal{F})$, then

$$
\left\|\mu_{1}-\mu_{2}\right\|=2 \sup _{B \in \mathcal{F}}\left|\mu_{1}(B)-\mu_{2}(B)\right| .
$$

In the sequel, we prove the following result.

Theorem. Let $P$ and $Q$ be transition probabilities from $S$ into $S$ and let

$$
\varepsilon=\sup _{s \in S}\|P(s, \cdot)-Q(s, \cdot)\|
$$

1991 Mathematics Subject Classification: Primary 60J10, 60J35; Secondary 93C40, 93E20.

Key words and phrases: Markov chains, transition probabilities, adaptive control, stochastic control. 
Then for $s, z \in S$ and $n \geq 1$ we have

$$
\left\|P^{n}(s, \cdot)-Q^{n}(z, \cdot)\right\| \leq \varepsilon\left(1+\beta+\ldots+\beta^{n-1}\right)+2 \beta^{n},
$$

where

$$
\beta=\frac{1}{2} \sup _{x, y \in S}\|P(x, \cdot)-P(y, \cdot)\| .
$$

REMARK 1. If $\varepsilon=0$, then (1) is exactly Ueno's inequality [9].

COROLlary 1. If $\beta<1$, then (1) implies that for $n$ sufficiently large we have

$$
\left\|P^{n}(s, \cdot)-Q^{n}(z, \cdot)\right\| \leq \frac{2 \varepsilon}{1-\beta}
$$

for each $s, z \in S$.

Suppose that $S$ is the state space for Markov chains having transition probabilities $P$ and $Q$ respectively. If there exists a probability measure $\pi_{P}$ on $(S, \mathcal{F})$ such that

$$
\sup _{s \in S}\left\|P^{n}(s, \cdot)-\pi_{P}(\cdot)\right\| \rightarrow 0 \quad \text { as } n \rightarrow \infty,
$$

at a geometric rate, then the Markov chain with transition probability $P$ is called uniformly ergodic and $\pi_{P}$ is the unique invariant probability measure for $P$.

Corollary 2. Let $\pi_{P}$ and $\pi_{Q}$ be the invariant probability measures for $P$ and $Q$ respectively. Assume that the Markov chains with transition probabilities $P$ and $Q$ are uniformly ergodic. If $\beta<1$, then

$$
\left\|\pi_{P}-\pi_{Q}\right\| \leq \frac{\varepsilon}{1-\beta} .
$$

It is well known that the Markov chain with transition probability $T$ is uniformly ergodic if and only if there exist a constant $c \in(0,1)$ and a positive integer $m$ such that

$$
\left\|T^{m}(s, \cdot)-T^{m}(z, \cdot)\right\| \leq 2 c
$$

for every $s, z \in S$. For a proof see, e.g., [2].

Put $P=T^{m}$ and fix a transition probability $Q$. Define

$$
\varepsilon=\sup _{s \in S}\left\|T^{m}(s, \cdot)-Q(s, \cdot)\right\| .
$$

Assume that (3) holds and consider $\beta$ defined by (2). Then $\beta<1$, and using Corollary 1 , we infer that for $n$ sufficiently large, we have

$\left\|Q^{n}(s, \cdot)-Q^{n}(z, \cdot)\right\| \leq\left\|Q^{n}(s, \cdot)-T^{m n}(s, \cdot)\right\|+\left\|T^{m n}(s, \cdot)-Q^{n}(z, \cdot)\right\| \leq \frac{4 \varepsilon}{1-\beta}$.

This enables us to state the following result. 
Corollary 3. If (3) holds and $2 \varepsilon /(1-\beta)<1$, then the Markov chain with transition probability $Q$ is also uniformly ergodic. Moreover,

$$
\left\|\pi_{T}-\pi_{Q}\right\| \leq \frac{\varepsilon}{1-\beta}
$$

where $\pi_{T}\left(\pi_{Q}\right)$ is the unique invariant probability measure for the transition probability $T(Q)$.

REMARK 2. Our main result and Corollaries 1-3 may have applications to approximation problems or adaptive control problems as studied in [3], [5], [6], [7] and [8]. A result closely related to Corollary 2 was proved by Stettner in [8], but our inequality (5) has a more elementary form. Also, our proof is quite elementary while the method of proof in [8] is based on the theory of bounded transition operators considered in [4]. However, Stettner's proof [8] can be used for studying some uniform convergence problems of $n$-step transition probabilities in different norms on the state space [6].

Proof of Theorem. We proceed by induction on $n$. It is easy to see that (1) holds for $n=1$. Suppose it holds for a positive integer $n$. Note that

$$
\begin{aligned}
\| P^{n+1}(s, \cdot) & -Q^{n+1}(z, \cdot) \| \\
& =\left\|P^{n} P(s, \cdot)-Q^{n} Q(z, \cdot)\right\| \\
& \leq\left\|P^{n} P(s, \cdot)-Q^{n} P(z, \cdot)\right\|+\left\|Q^{n} P(z, \cdot)-Q^{n} Q(z, \cdot)\right\| \\
& \leq\left\|P^{n} P(s, \cdot)-Q^{n} P(z, \cdot)\right\|+\varepsilon .
\end{aligned}
$$

Moreover, we have

$$
\left\|P^{n} P(s, \cdot)-Q^{n} P(z, \cdot)\right\|=2 \sup _{B \in \mathcal{F}}|L(B)|,
$$

where

$$
L(B)=\int_{S} P(x, B) \mu(s, z)(d x)
$$

for any $B \in \mathcal{F}$ and $\mu(s, z)(\cdot)=P^{n}(s, \cdot)-Q^{n}(z, \cdot)$.

Define

$$
\varphi(x)=P(x, B)-\inf _{y \in S} P(y, B) .
$$

Note that $\varphi \geq 0$ on $S$ and

$$
L(B)=\int_{S} \varphi(x) \mu(s, z)(d x) .
$$

Fix $B \in \mathcal{F}$. Without loss of generality, we can assume that $|L(B)|=$ $L(B)$ (otherwise, use $-\mu(s, z)(d x)$ instead of $\mu(s, z)(d x))$. By the Hahn decomposition theorem [1], there exists a set $D \in \mathcal{F}$ such that

$$
\begin{array}{ll}
\mu(s, z)(E) \geq 0 & \text { for all } E \in \mathcal{F}, E \subset D, \\
\mu(s, z)(E) \leq 0 & \text { for all } E \in \mathcal{F}, E \subset S \backslash D .
\end{array}
$$


Note that

$$
\begin{aligned}
|L(B)|=L(B) & =\int_{D} \varphi(x) \mu(s, z)(d x)+\int_{S \backslash D} \varphi(x) \mu(s, z)(d x) \\
& \leq \int_{D} \varphi(x) \mu(s, z)(d x) \leq \mu(s, z)(D) \sup _{x \in S} \varphi(x) \\
& \leq \frac{1}{2} \mu(s, z)(D) \sup _{x, y \in S} 2|P(x, B)-P(y, B)| .
\end{aligned}
$$

Hence,

$$
L(B) \leq \mu(s, z)(D) \cdot \frac{1}{2} \sup _{x, y \in S}\|P(x, \cdot)-P(y, \cdot)\|=\mu(s, z)(D) \cdot \beta .
$$

But

$$
\begin{aligned}
\mu(s, z)(D) & =P^{n}(s, D)-Q^{n}(z, D) \leq \frac{1}{2} 2 \sup _{F \in \mathcal{F}}\left|P^{n}(s, F)-Q^{n}(z, F)\right| \\
& =\frac{1}{2}\left\|P^{n}(s, \cdot)-Q^{n}(z, \cdot)\right\| .
\end{aligned}
$$

This and (7) imply that

$$
|L(B)|=L(B) \leq \frac{1}{2}\left\|P^{n}(s, \cdot)-Q^{n}(z, \cdot)\right\| \cdot \beta .
$$

By (6) and (8) we obtain

$$
\left\|P^{n} P(s, \cdot)-Q^{n} P(z, \cdot)\right\| \leq\left\|P^{n}(s, \cdot)-Q^{n}(z, \cdot)\right\| \cdot \beta .
$$

Applying this inequality, (5) and our induction hypothesis we finally get

$$
\begin{aligned}
\left\|P^{n+1}(s, \cdot)-Q^{n+1}(z, \cdot)\right\| & \leq \varepsilon+\left\|P^{n}(s, \cdot)-Q^{n}(z, \cdot)\right\| \cdot \beta \\
& \leq \varepsilon+\beta\left(\varepsilon+\varepsilon \beta+\ldots+\varepsilon \beta^{n-1}+2 \beta^{n}\right) \\
& =\varepsilon\left(1+\beta+\ldots+\beta^{n}\right)+2 \beta^{n+1},
\end{aligned}
$$

which we wanted to prove.

\section{References}

[1] R. B. Ash, Real Analysis and Probability, Academic Press, New York, 1972.

[2] J. P. Georgin, Contrôle de chaînes de Markov sur des espaces arbitraires, Ann. Inst. H. Poincaré Sér. B 14 (1978), 255-277.

[3] O. Hernandez-Lerma, Adaptive Markov Control Processes, Springer, New York, 1989.

[4] N. W. Kartashov, Criteria for uniform ergodicity and strong stability of Markov chains in general state space, Probab. Theory Math. Statist. 30 (1984), 65-81.

[5] G. B. Di Masi and E. Stettner, Bayesian ergodic adaptive control of discrete time Markov processes, Stochastics and Stochastics Reports 54 (1995), 301-316.

[6] A. S. Nowak and E. Alt man, $\varepsilon$-Nash equilibria in stochastic games with uncountable state space and unbounded cost, Technical Report, Institute of Mathematics, Wrocław University of Technology, 1998. 
[7] W. J. Runggaldier and E. Stettner, Approximations of Discrete Time Partially Observed Control Problems, Appl. Math. Monographs 6, C.N.R., Pisa, 1994.

[8] E. Stettner, On nearly self-optimizing strategies for a discrete-time uniformly ergodic adaptive model, Appl. Math. Optim. 27 (1993), 161-177.

[9] T. Ueno, Some limit theorems for temporally discrete Markov processes, J. Fac. Sci. Univ. Tokyo 7 (1957), 449-462.

Andrzej S. Nowak

Institute of Mathematics

Wrocław University of Technology

Wybrzeże Wyspiańskiego 27

50-370 Wrocław, Poland

E-mail: nowak@im.pwr.wroc.pl

Received on 9.12.1996;

revised version on 15.12.1997 